\title{
Emission from Mesoscopic-Size Islands Formed in a GaAs/AlAs Double Layer Structure
}

\author{
A. WysmoleK ${ }^{a, b, *}$, B. ChWalisz ${ }^{a, b}$, M. Potemski $^{b}$, \\ R. StȩPNiEWski ${ }^{a}, \mathrm{~A}$. BABIŃSKI ${ }^{a, b}, \mathrm{~S} \cdot \mathrm{RAYMOND}^{c}$ \\ AND V. THIERRY-MIEG ${ }^{d}$
}

${ }^{a}$ Institute of Experimental Physics, Warsaw University, Hoża 69, Warsaw, Poland ${ }^{b}$ Grenoble High Magnetic Field Laboratory, MPI/FKF and CNRS

Grenoble, France

${ }^{c}$ Institute for Microstructural Sciences, NRC, Ottawa, Canada, K1A 0R6

${ }^{d}$ Laboratoire de Photonique et de Nanostructures, CNRS

91460 Marcoussis, France

\begin{abstract}
The nature of sharp emission lines which are present in macro-luminescence experiments on a type-II GaAs/AlAs double quantum well structure is discussed. The experiments, which also include micro-luminescence measurements, allowed us to conclude that the sharp emission lines observed originate from lateral GaAlAs islands of a few $\mu \mathrm{m}$ in diameter. They serve as efficient type-I recombination centers for indirect excitons and/or carriers which diffuse in the GaAs/AlAs QW structure and strongly affect the emission processes observed in macro-luminescence experiments. These traps can easily be filled with electron-hole pairs, giving rise to the formation of neutral excitons as well as more complex excitonic molecules. Magnetoluminescence spectra from single islands resemble those observed for natural quantum dots formed in narrow GaAs quantum wells.
\end{abstract}

PACS numbers: 78.67.De, 78.20.Ls, 71.35.Lk, 73.20.-r, 78.47.+p

\section{Introduction}

The quest for the possibility of optical generation of cold, tunable-density, two-dimensional electron-hole gas has stimulated investigations of double quan-

*corresponding author; e-mail: Andrzej.Wysmolek@fuw.edu.pl 
tum well structures such as, for example, GaAs/AlAs hetero-wells with a built in type-II interface. Luminescence spectra of such systems are known to exhibit a number of intriguing properties which have been interpreted as observation of a precursor of the exciton condensate [1]. Alternatively, they are assigned to effects of trapping of photoexcited carriers in naturally built-in quantum-dot like objects formed by potential fluctuations caused by the interface roughness [2, 3].

Recently we have shown that the broad, indirect macro-luminescence of a GaAs/AlAs double quantum well structure can also be accompanied by a spectrum of sharp emission lines spreading up to $150 \mathrm{meV}$ below the characteristic energy of the direct $\Gamma-\Gamma$ transition [4]. The appearance of these lines was not well understood, though they were tentatively attributed to laterally confined type-II quantum dot like objects [4]. In order to explain the nature of this intriguing emission, we have employed several experimental techniques, including time-resolved photoluminescence, micro-photoluminescence mapping as well as macro- and micro-luminescence experiments in high magnetic fields. The results obtained allowed us to suggest that these sharp emission lines originate from GaAlAs islands which replace the AlAs layer, and which are a few $\mu \mathrm{m}$ in lateral size. These islands serve as efficient type-I recombination centers for indirect excitons and/or carriers which diffuse in the GaAs/AlAs QW structure. They can easily be filled with electron-hole pairs giving rise to the formation of neutral excitons as well as more complex excitonic molecules.

\section{Experimental details}

The experiments were performed at different temperatures, in the range from $1.3 \mathrm{~K}$ up to $110 \mathrm{~K}$, in magnetic fields up to $28 \mathrm{~T}$ applied either perpendicular or along the two-dimensional plane of the structure. For above barrier excitation, a $514 \mathrm{~nm}(2.4 \mathrm{eV}) \mathrm{Ar}^{+}$laser line was used. Photoluminescence excitation experiments were carried out using Ti-sapphire laser, in a tunable range of 1.7-1.8 eV. Continuous-wave $(\mathrm{cw})$ spectra were recorded using a single grating spectrometer $(0.05 \mathrm{meV}$ resolution) equipped with CCD detector. Time-resolved measurements were performed using a fast optical chopper (10 ns resolution) and a standard photon-counting system reading the signal from the GaAs-photomultiplier attached to a $0.5 \mathrm{~m}$ long, single grating monochromator.

The zero field micro-photoluminescence ( $\mu$-PL) mapping experiments were performed using a standard $\mu$-PL system with the microscope objective providing a spatial resolution of about $5 \mu \mathrm{m}$. For the $\mu$-PL experiments in magnetic fields a two-fiber optical system was used. The sample was excited with a $514 \mathrm{~nm} \mathrm{Ar}^{+}$ laser line using a single-mode fiber coupled to a two-lens system providing an excitation spot on the sample of about $5 \mu \mathrm{m}$ in diameter. The emission from the sample was collected via a $600 \mu \mathrm{m}$ fiber. 


\section{Results and discussion}

\subsection{Macro-luminescence - zero-field characteristics}

The active part of the structure used for the experiments consists of a $2.4 \mathrm{~nm}$ wide GaAs layer and $10 \mathrm{~nm}$ wide $\mathrm{AlAs}$ layer embedded in $\mathrm{Ga}_{0.67} \mathrm{Al}_{0.33}$ As barriers (see the inset in Fig. 1). Characteristic cw photoluminescence (PL) spectra of this structure measured under very low excitation power $\left(\sim 0.1 \mathrm{~mW} / \mathrm{cm}^{2}\right)$ in zero field and at $28 \mathrm{~T}$ are presented in Fig. 1. The zero-field spectrum consists of a weak emission peak which corresponds to the direct $\Gamma_{\mathrm{GaAs}}-\Gamma_{\mathrm{GaAs}}$ recombination and four, more pronounced lines associated with indirect $X_{\mathrm{AlAs}}-\Gamma_{\mathrm{GaAs}}$ transitions: a zero-phonon line and its $T A(X)_{\mathrm{GaAs}}, L O(X)_{\mathrm{GaAs}}$, and $L O(X)_{\mathrm{AlAs}}$ phonon replicas [4]. Such a spectrum is characteristic of type-II GaAs/AlAs systems for which the lowest electronic state in the AlAs well is of $X_{X Y}$ symmetry and the $X_{Z}$ state is located a few meV higher. The observed ordering of $X_{X Y}$ and $X_{Z}$ states is a subtle result of the interplay between the confinement and strain $[5,6]$.

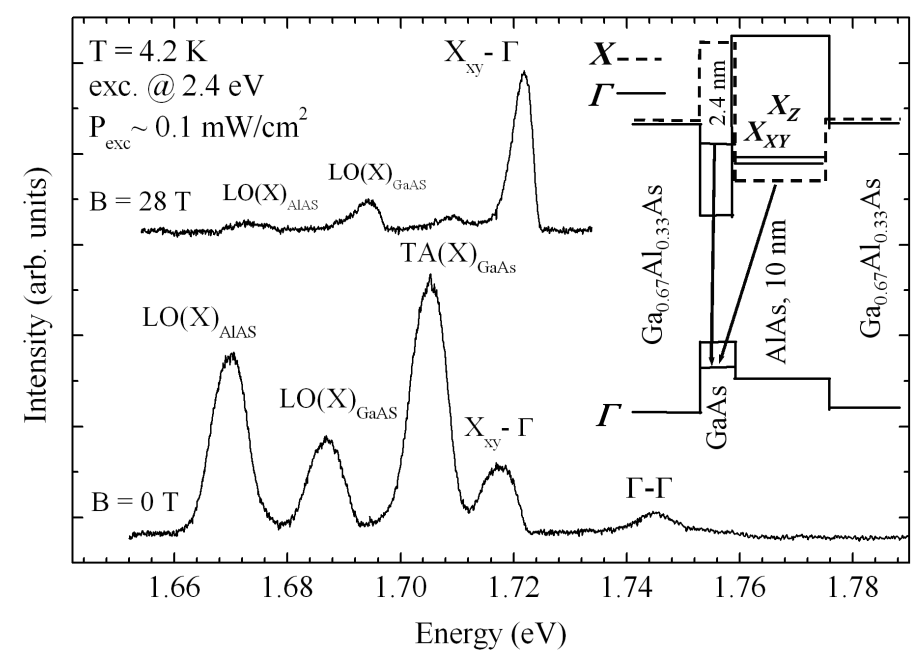

Fig. 1. The diagram of the investigated structure and its representative PL spectra under low excitation power $\left(\sim 0.1 \mathrm{~mW} / \mathrm{cm}^{2}\right)$ for $B=0 \mathrm{~T}$ and $28 \mathrm{~T}$.

The spectra change considerably under higher excitation intensities. As can be seen in Fig. 2, for excitation density of a few $\mathrm{mW} / \mathrm{cm}^{2}$, the zero-field spectra show an ensemble of numerous sharp emission lines, with typical half-widths less than $0.1 \mathrm{meV}$. These lines are observed over a very broad spectral range $(1.58 \mathrm{eV}-$ $1.72 \mathrm{eV}$ ). At zero-magnetic field, the sharp lines are mostly grouped around $1.6 \mathrm{eV}$, forming a band which we refer to as the "S-band".

As shown in the inset of Fig. 2, the PL-excitation spectra of the direct $\Gamma-\Gamma_{\mathrm{hh}}$ emission, involving heavy hole (hh) valence states in the GaAs QW, as well as of indirect $X_{Z}-\Gamma, X_{X Y}-\Gamma$ transitions including their phonon replicas 


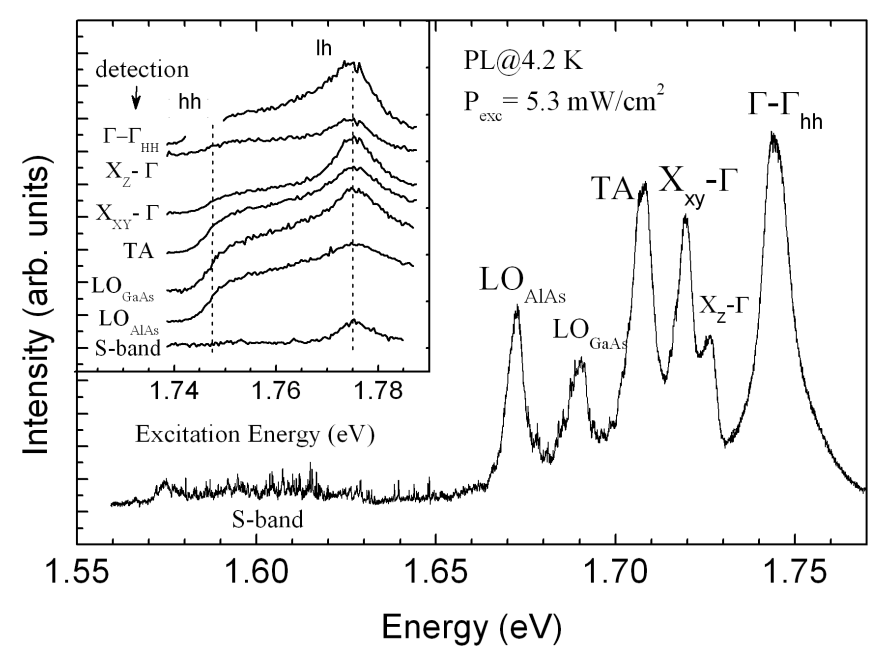

Fig. 2. Photoluminescence spectrum of the $1 \mathrm{~mm}$ size area of the sample measured at $T=4.2 \mathrm{~K}$ with $5.3 \mathrm{~mW} / \mathrm{cm}^{2}$ power density. PL-excitation spectra of different emission bands are shown in the inset.

show a characteristic absorption onset coinciding with the $\Gamma-\Gamma_{\mathrm{hh}}$ luminescence line and a pronounced peak around $1.775 \mathrm{eV}$. The latter peak is attributed to the direct absorption transition in the GaAs QW, which however involves the light-hole (lh) valence band subband. The absorption onset in the vicinity of the fundamental $\Gamma-\Gamma_{\mathrm{hh}}$ transition in the GaAs QW is somehow less pronounced in the PL-excitation spectrum of the $S$-band but the lh peak remains clearly visible. In general, however, all measured PL-excitation spectra are quite similar in shape and we think therefore that the entire PL spectrum observed in our experiments originates from the same active part of the sample.

The overall shape of the measured PL-excitation (PLE) spectra (less pronounced hh transition but enhanced lh peak) is however intriguing. As a matter of fact they resemble the PLE-spectra expected for $p$-type modulation quantum wells in which the heavy hole subband is populated with holes and therefore the fundamental $\Gamma-\Gamma_{\mathrm{hh}}$ absorption is quenched, but $\Gamma-\Gamma_{\mathrm{lh}}$ can be strong and even show an excitonic enhancement [7]. Our structure is not intentionally doped but we believe that the heavy hole subband in the GaAs well can be populated efficiently under optical excitation since, as discussed later, the characteristic PL decay times are observed to be as long as $1 \mathrm{~ms}$. Therefore, the observed shape of the PL-excitation spectra may result from a dynamical effect of the efficient filling of the heavy hole subband once the laser energy is tuned over the otherwise strong $\Gamma-\Gamma_{\mathrm{hh}}$ absorption resonance. We think this could lead to quenching of the $\Gamma-\Gamma_{\mathrm{hh}}$ resonance in PL-excitation experiments and the appearance of a kind of Moss-Burstein effect. However, more PL-excitation measurements, with a wide range of laser power, are needed to confirm this idea. 


\subsection{Temperature dependence of the macro-luminescence}

Photoluminescence spectra measured at temperatures between 4.7 and $104 \mathrm{~K}$ are shown in Fig. 3. It is clearly seen that the broad emission lines attributed to the recombination of indirect excitons disappear from the PL spectrum below $30 \mathrm{~K}$. This result suggests that the emission identified as due to indirect excitons involves mostly indirect excitons localized at fluctuations of the GaAs/AlAs interface. On the other hand, temperature is not a critical parameter for observation of the sharp lines. Some of them even gain in intensity with temperature in the low temperature range. Certain sharp lines can be followed up to temperatures as high as $60 \mathrm{~K}$. The absolute intensity of the $S$-band increases with increasing temperature, reaches a maximum around $40 \mathrm{~K}$ then decreases, and finally disappears from the spectrum above $110 \mathrm{~K}$. The appearance of sharp lines is clearly not a low temperature phenomenon and also it can hardly be explained in terms of localization effects at the GaAs/AlAs interface, simply induced by GaAs/AlAs well-width fluctuations.

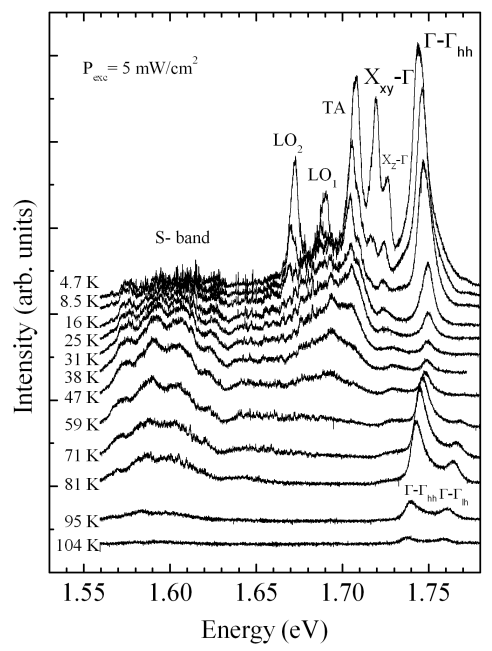

Fig. 3. Photoluminescence spectra measured for different temperatures between 4.7 and $104 \mathrm{~K}$.

Very probably, there exists another type of "localization trap" in the active part of the sample which leads to the emergence of the observed sharp emission lines. The density of these traps must be quite low as resolved sharp lines are easily observed in macro-luminescence experiments. On the other hand, the characteristic localization energy associated with the "traps" must be quite large, since the sharp emission lines spread over a wide spectral range and the lines are visible at rather high temperatures. To account for the appearance of such "deep traps" in our sample, we note the possibility of effective Ga diffusion into the AlAs layer during the MBE growth of the structure. This diffusion, if laterally inhomogeneous, 
could lead to the formation of islands, where the perfect double GaAs/AlAs layer is replaced by a kind of GaAs-GaAlAs quantum well layer embedded in between regular GaAlAs barriers. Such islands could serve as efficient type-I recombination centers for nearby long lived carriers (confined in the X-conduction band minimum of the AlAs layer and $\Gamma$-valence band maximum in the GaAs layer). The proposed scenario implies that the diffusion process of the long-lived carriers is an important element in understanding the observed phenomena and should be reflected in the kinetics of the emission. This picture should also be confirmed in micro-PL measurements. The results of such experiments are reported in the following sections.

\subsection{The time-characteristics of the system}

Time-resolved measurements were performed in a way that exciting light was formed into a rectangular pulse train with time period $T=2 \mathrm{~ms}$ and $50 \%$ duty cycle. The first $1 \mathrm{~ms}$ of the period was long enough to achieve a steady state of the system. Then, the exciting light was switched off within $10 \mathrm{~ns}$ and the PL transients were observed. The fastest decay times observed in the emission at $T=4.2 \mathrm{~K}$ are associated with direct $\Gamma-\Gamma$ recombination in the GaAs QW and the $X_{Z}-\Gamma$ transitions involving electrons from the AlAs QW and holes from the GaAs QW (Fig. 4). They are shorter than $10 \mathrm{~ns}$ which is the limit of our experiment. As can be seen in Fig. 4, the transients observed for the $X_{X Y}$ emission and its phonon replicas cannot be described by a mono-exponential dependence. Just after switching off the excitation pulse, the emission intensity decays fast (microsecond scale) but later a much slower decay is observed (millisecond range). The longest decay time, of about $1 \mathrm{~ms}$, is seen for the $X_{X Y}$ transition, for times

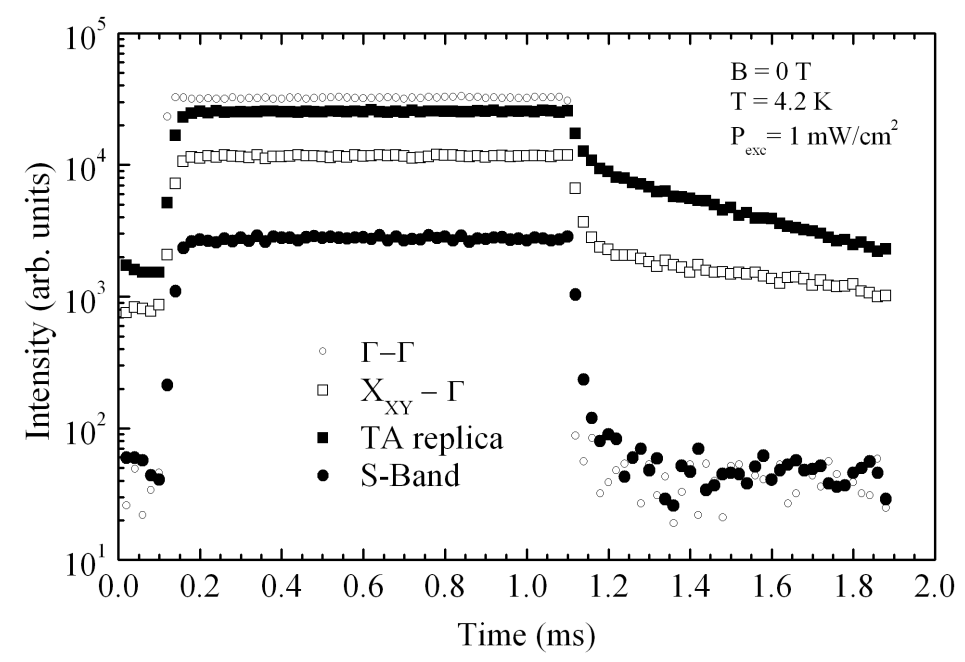

Fig. 4. Time-resolved emission measured for different transitions in zero field with a time window of $\Delta t=20 \mu \mathrm{s}$. 
sufficiently long after the excitation pulse. The phonon replicas of the $X_{X Y}$ emission show shorter decay times of about $0.5 \mathrm{~ms}$. The fast decay process is more pronounced when the excitation intensity is increased. On the other hand, a few microseconds after switching off the laser the PL intensity is independent of the excitation power (see Fig. 5). For the lowest excitation powers, only a slow decay component is observed. The characteristic decay time detected for the $S$-band is in the $\mu$ s range and decreases with increasing excitation power. The observed behavior could be qualitatively reproduced using a simplified model taking into
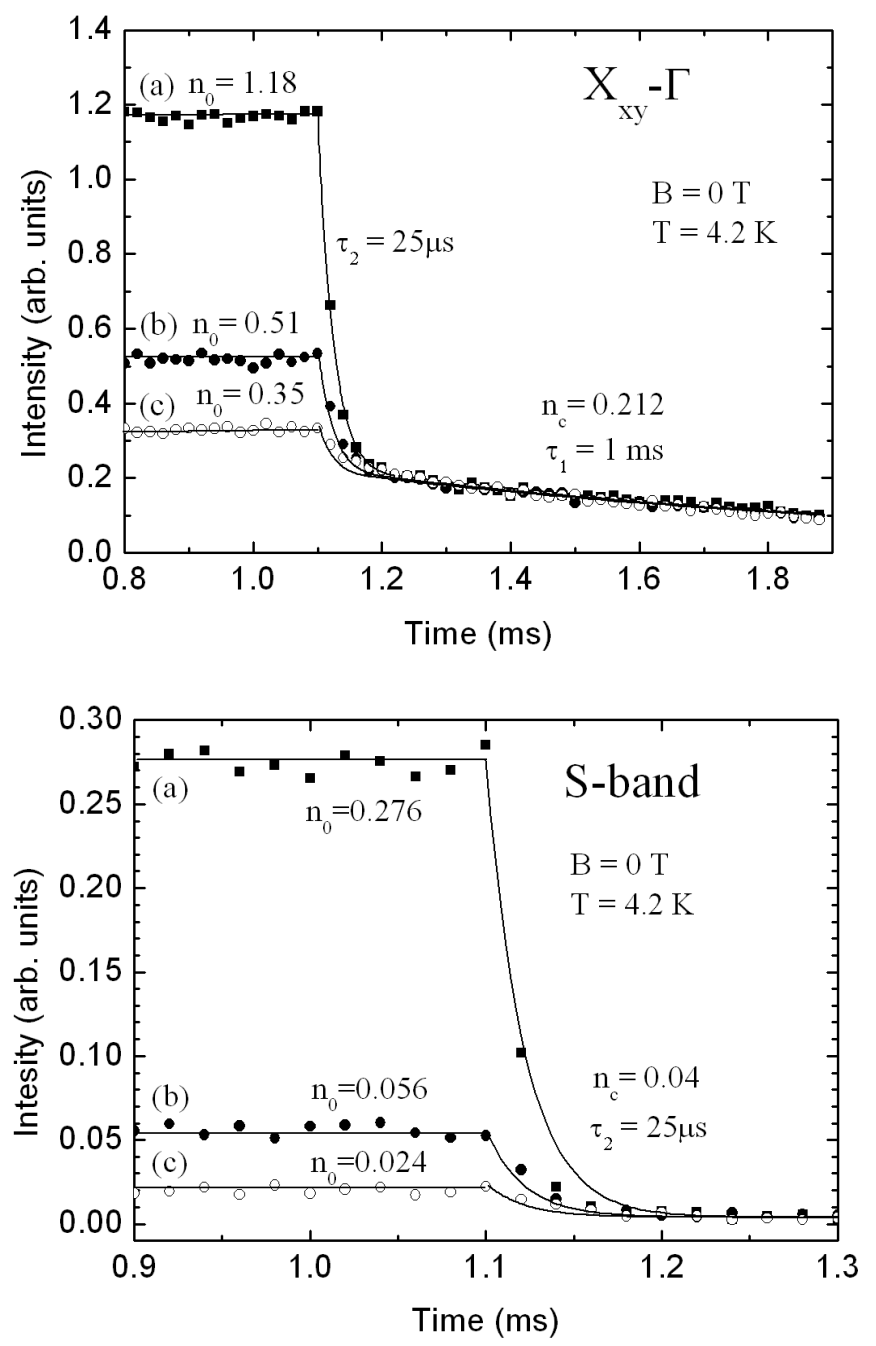

Fig. 5. The luminescence transients measured at $T=4.2 \mathrm{~K}$ for the $X_{X Y}-\Gamma$ transition (top panel) and for the $S$-band (bottom panel). The experimental data $(a),(b)$, and $(c)$ correspond to the average excitation power set to $1,0.3,0.12 \mathrm{~mW} / \mathrm{cm}^{2}$, respectively. 
account the interplay between diffusion and localization of carriers and/or indirect excitons. We assume that in the case of low excitation the photo-generated carriers are effectively trapped at interface fluctuations and recombine with a long decay time $\tau_{1}$ (in the ms range). This long decay time results from the small overlap of the electron and hole wave-functions as well as from $k$-selection rules. The number of such traps is limited, hence by increasing excitation power one can achieve a certain critical concentration $n_{\mathrm{c}}$, for which this recombination channel is not sufficient and the excess excitons (carriers) begin to diffuse "searching" for other "recombination possibilities". This "escape" process can be described by a characteristic time $\tau_{2}$ assumed to be in the $\mu$ s range. Such reasoning can be expressed by the following set of differential equations:

$$
\frac{\mathrm{d} n}{\mathrm{~d} t}=\left\{\begin{array}{ll}
-\frac{n}{\tau_{1}}-\frac{n-n_{c}}{\tau_{2}}+G(t) & \text { for } n \geq n_{\mathrm{c}} \\
-\frac{n}{\tau_{1}} & \text { for } n \leq n_{\mathrm{c}}
\end{array},\right.
$$

where $G(t)$ is the generation function $\left(G(t)=G_{0}\right.$ if $t<0$ and $G(t)=0$ when $t>0)$. Equation (1) has a simple solution for $t>0$ :

$$
n(t)=\left\{\begin{array}{ll}
\left(n_{0}-\frac{\tau_{1}}{\tau_{1}+\tau_{2}} n_{\mathrm{c}}\right) \exp \left(-\left(\frac{1}{\tau_{1}}+\frac{1}{\tau_{2}}\right) t\right)+\frac{\tau_{1}}{\tau_{1}+\tau_{2}} n_{\mathrm{c}} & \text { for } n \geq n_{\mathrm{c}} \\
n_{\mathrm{c}} \exp \left(-\frac{1}{\tau_{1}}\left(t-t_{\mathrm{c}}\right)\right) & \text { for } n \leq n_{\mathrm{c}}
\end{array},\right.
$$

where $n_{0}$ is the initial concentration of excitons (carriers) and $t_{\mathrm{c}}=$ $\frac{\tau_{1} \tau_{2}}{\tau_{1}+\tau_{2}} \ln \left(\left(1+\frac{\tau_{1}}{\tau_{2}}\right) \frac{n_{0}}{n_{c}}-\frac{\tau_{1}}{\tau_{2}}\right)$ accounts for critical time resulting from the condition $n\left(t_{\mathrm{c}}\right)=n_{\mathrm{c}}$.

The above equation reflects fairly well the observed decay of the indirect emission lines. It should be noted that for a given emission band, the same set of parameters $\tau_{1}, \tau_{2}$, and $n_{\mathrm{c}}$ can be successfully used to describe the decays measured for different excitation powers. The only parameter which depends on the excitation power is the initial concentration of electron-hole pairs $n_{0}$. In Fig. 5 , the experimental results obtained for $X_{X Y}-\Gamma$ transitions are plotted, together with the calculated curves obtained with the following set of parameters: $\tau_{1}=1 \mathrm{~ms}$, $\tau_{2}=25 \mu$ s and critical concentration $n_{\mathrm{c}}=0.212$. The calculated curves presented in Fig. 5 for the $S$-band were obtained in a similar way assuming $n_{\mathrm{c}}=0.04$. The initial concentration $n_{0}$ was set to be equal to the initial PL intensity and is found to be a linear function of the excitation power. In spite of the fact that the model presented is very simple it provides an excellent description of the experimental data. It proves that diffusion of the indirect excitons (carriers) is a crucial element determining time characteristics of both the indirect transitions and the sharp emission lines.

\subsection{Macro-luminescence - magnetic field behavior}

The emergence of sharp emission lines can be enhanced by the application of the magnetic field in the direction perpendicular to the QW plane $\left(B_{\perp}\right)$. However, 
it is noteworthy that the sharp lines do not appear in the spectrum, even at $28 \mathrm{~T}$, when the excitation power is set bellow some critical value (see Fig. 1). The only visible effect in this case is a magnetic field induced the quenching of the emission related to the recombination of indirect excitons. This effect has been reported previously and explained as due to localization of photo-excited carriers in type-II potential fluctuations at the GaAs/AlAs interface [3]. It is argued that the application of $B_{\perp}$ induces an additional lateral separation of the electron and hole
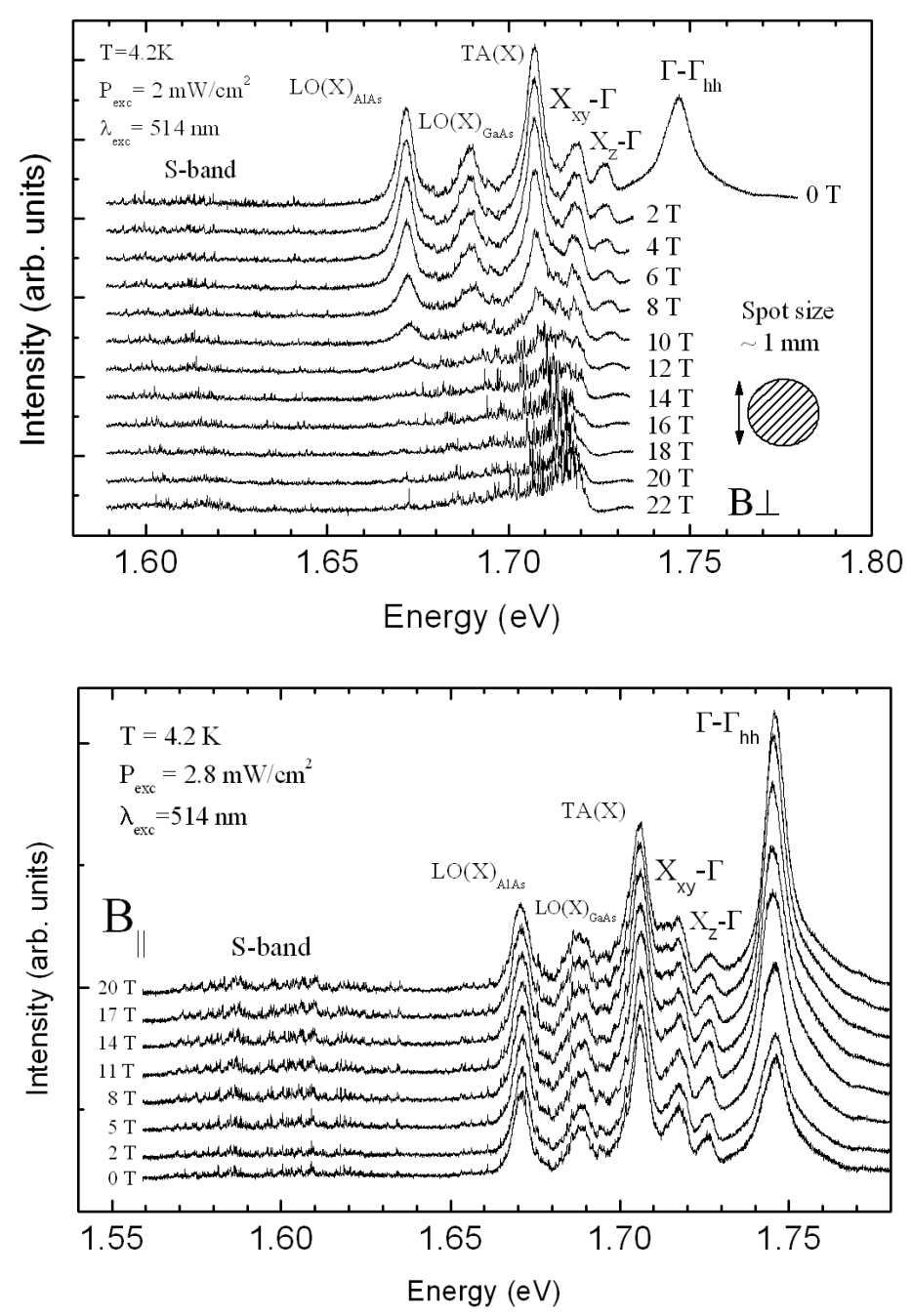

Fig. 6. Macro-PL spectra measured for magnetic field perpendicular to the QW plane in the range $0-22 \mathrm{~T}$ with excitation power $\sim 2 \mathrm{~mW} / \mathrm{cm}^{2}$ (top panel). Macro-PL spectra measured for in-plane magnetic field between 0 and $20 \mathrm{~T}$ with excitation power of $2.8 \mathrm{~mW} / \mathrm{cm}^{2}$ (bottom panel). 


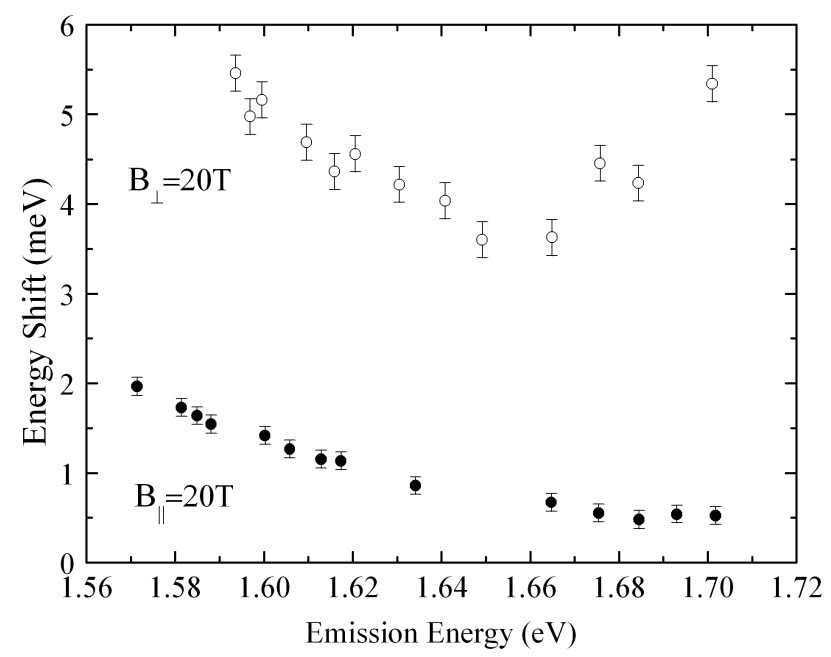

Fig. 7. Energy shifts measured for different sharp lines for the magnetic field direction perpendicular to the quantum well plane $\left(B_{\perp}\right)$ and for the magnetic field direction parallel to the quantum well plane $\left(B_{\|}\right)$.

wavefunctions which leads to a decrease in the emission intensity corresponding to the indirect exciton recombination. In the case when the excitation power is set beyond the critical value, the application of high $B_{\perp}$ transforms the broad indirect spectrum into a forest of sharp lines (Fig. 6). On the other hand, the application of the magnetic field in the direction parallel to the QW plane does not affect the PL spectrum significantly (Fig. 6). The energy shifts registered for different sharp emission lines at $20 \mathrm{~T}$ for $B_{\perp}$ are a few times larger than those observed for $B_{\|}$(Fig. 7). Such an effect can be explained assuming that the observed energy changes correspond to the differences in diamagnetic shifts for $B_{\perp}$ and $B_{\|}$ configurations. Since the diamagnetic shift is expected to be larger for spatially delocalized objects it could be concluded that the sharp lines originate from objects characterized by large $x y$ - and small $z$-dimensions [8]. As can be seen in Fig. 7, diamagnetic shifts observed for in-plane magnetic fields show a monotonic decrease with increasing emission energy. This suggests that the sharp emission lines observed at lower energies originate from objects of larger $z$-dimensions. On the other hand, the diamagnetic shifts observed for $B_{\perp}$ range between 3.5 and $6 \mathrm{meV}$. The results are in contradiction to the intuitive explanation that more localized states should reveal smaller diamagnetic shifts. On the other hand, the observed change in the diamagnetic shift is consistent with the hypothesis that our sharp lines originate from GaAlAs islands formed in the vicinity of the GaAs/AlAs interface. In this case the objects with a larger Ga content give rise to the emission at lower energies, and that is not contradictory to the larger value of the diamagnetic shift which is determined mostly by island dimensions. 


\subsection{Micro-luminescence experiments}

A further understanding of the properties of our system is provided by $\mu$-PL experiments. Mapping the PL with $\sim 5 \mu \mathrm{m}$ resolution we find that the sharp lines originate from well-isolated islands. The representative $\mu$-PL spectrum of an isolated "spiky" island is presented in Fig. 8. As shown in Fig. 9 such an

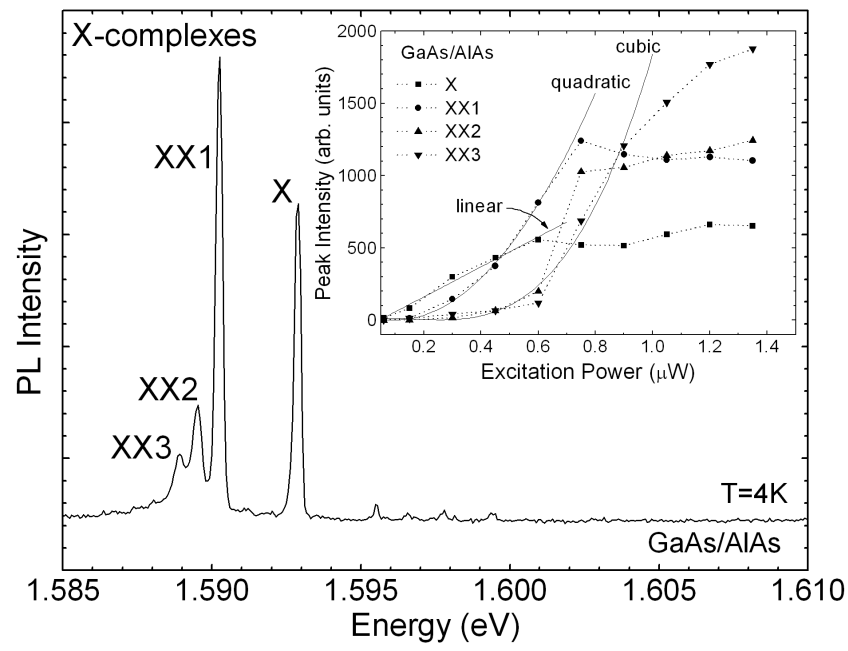

Fig. 8. Photoluminescence spectrum of a single mesoscopic object contributing to the $S$-band. The inset - PL intensity versus excitation power.

island might be well isolated in a region as large as of $60 \times 60$ micrometer square. We observe that the islands of "spiky" emissions are randomly distributed on the sample surface with a density of about $10^{5} \mathrm{~cm}^{-2}$. At the same time the intensity map of the broad indirect emission lines is observed to be homogeneous (within our spatial resolution). For very low excitations only a single $X$ emission line is observed. With increasing excitation power an additional line at an energy of about $2.7 \mathrm{meV}$ below the initial emission appears (the $X X 1$ line in Fig. 8). Such a doublet structure is similar to that observed in the micro-luminescence of natural GaAs and InAs quantum dots [9-14], and can be assigned to neutral $(X)$ and charged excitons and/or biexcitons $(X X 1)$. A further increase in the excitation power leads to the appearance of additional low energy lines which we attribute to the formation of more complex excitonic molecules (lines $X X 2$ and $X X 3)$. The inset in Fig. 8 illustrates the excitation power dependences of the PL intensity. In the limit of low excitation the intensity of the $X$ line, attributed to the neutral exciton recombination, varies linearly with increasing excitation power. Quadratic and cubic dependences were observed for the $X X 1$ and $X X 3$ lines, respectively. Such behavior was already observed for GaAs QDs [9, 14] and per analogy, the excitonic emission can be attributed to the recombination of the 

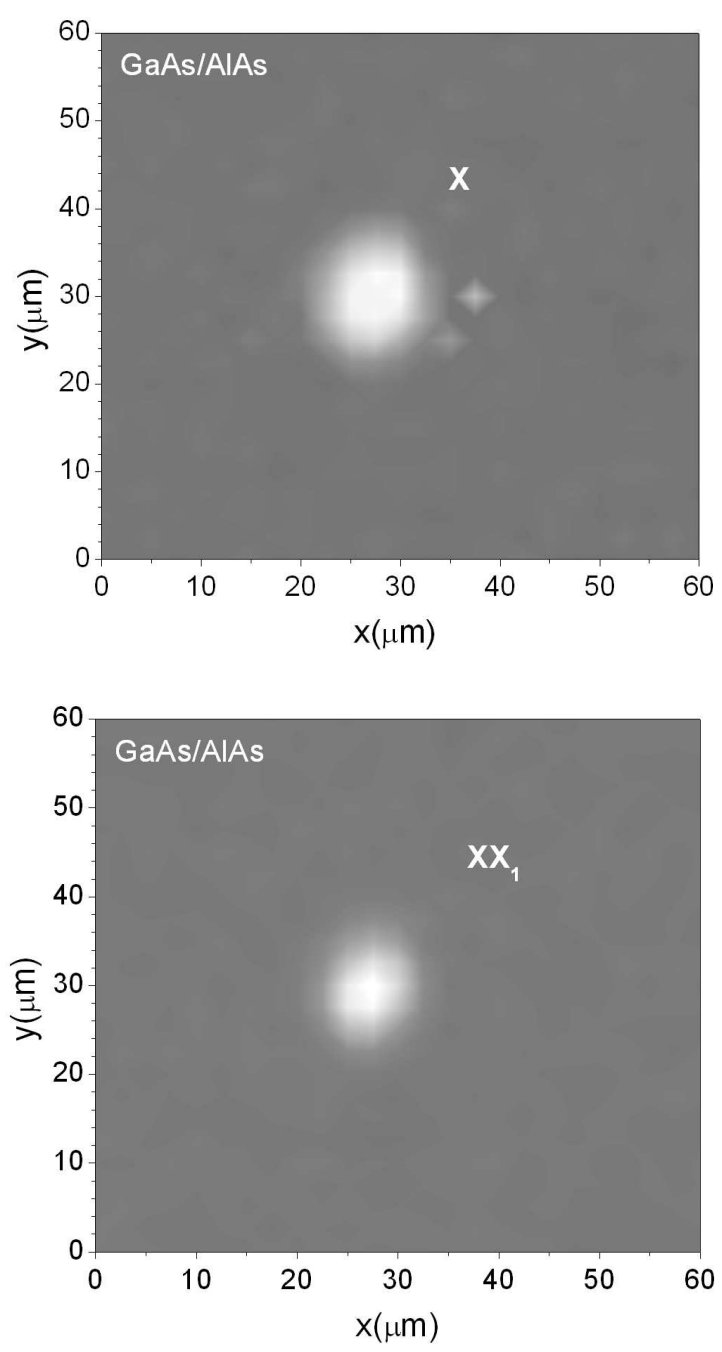

Fig. 9. $\mu$-PL maps measured for $X$ (top panel) and $X X 1$ emission (bottom panel).

neutral exciton, biexciton, tri-exciton, and higher order excitonic complexes [15]. In the high excitation regime the subsequent emissions saturate when the emissions of more complex structures gain in intensity.

The application of magnetic field allows us to verify the identification of the observed transitions. Figure 10 illustrates the typical magnetic field behavior of the emission of a selected single object. All emission lines show a significant and very similar diamagnetic shift. The $X$ line attributed to the neutral exciton splits into two well-resolved Zeeman components. The effective $g$-factor of the observed transitions varies from object to object, from approx. 0.9 to 1.2. The magnetic field behavior of the low energy part of the spectrum, related to other excitonic 


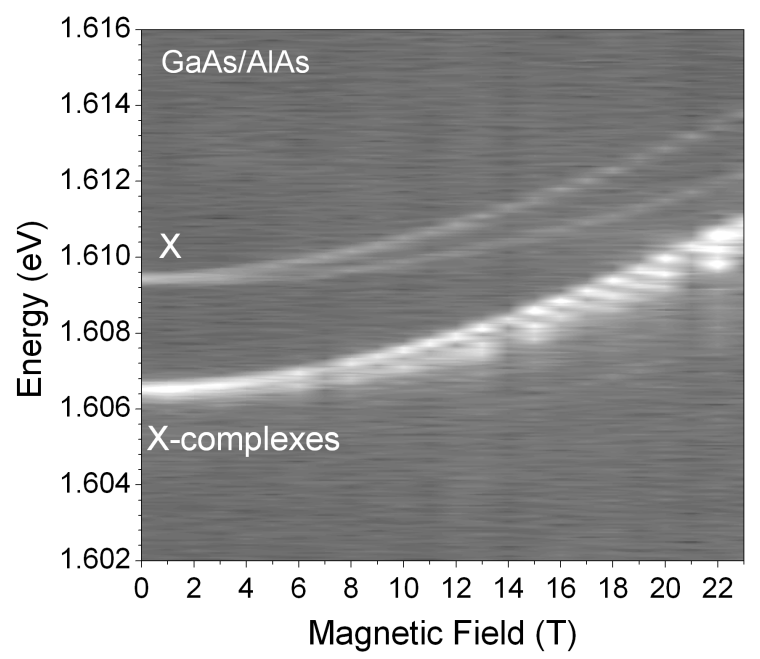

Fig. 10. Map showing magnetic field evolution (up to $23 \mathrm{~T}$ ) of the micro-PL spectra of a single GaAs/AlAs island.

complexes, is more complicated. They split into several components. Some of them gain in intensity in a strong magnetic field and finally become dominant in the spectrum. It was observed that the highest energy component of the $X$ complex lines ( $X X 1$ emission) also splits with an effective $g$-factor similar to the one observed for the $X$ line. This supports its identification as biexciton emission. However, the appearance of charged exciton lines cannot be completely ruled out as they may coincide with the biexciton emission and show a similar Zeeman pattern. More work is under way to identify better the particular excitonic complexes. It is remarkable however that the observed $\mu$-magneto-PL behavior is reproducible when studying various parts of the sample and in a broad range of the emission energy (from $1.58 \mathrm{eV}$ to $1.66 \mathrm{eV}$ ).

\subsection{Nature of the objects responsible for sharp emission lines}

The results obtained including both macro- and $\mu$-PL are difficult to explain using a simple model of a fluctuating GaAs/AlAs interface. One could expect that monolayer well-width fluctuations can provide a localization energy in the range of $\mathrm{meV}[11,16,17]$, but they are not sufficient to explain the observed red-shifts of the order of $100 \mathrm{meV}$. However, the results can be understood when the diffusion of Ga into the AlAs part of the QW is taken into account [18-20]. This would cause the formation of mesoscopic GaAlAs objects with an energy band gap below the $X$ state. For gallium composition below 0.3 we expect that the islands will no longer behave like indirect objects but will serve as centers of direct recombination which capture diffusing excitons. One can expect that the formation of such islands can be favored in the vicinity of extended defects such as dislocation loops, intrinsic point [18] and/or oval defects with enhanced concentration of gallium [21, 22]. 
The dimensions of oval defects are of order several $\mu \mathrm{m}[21,22]$, thus very similar to the size of the islands determined from the $\mu$-PL mapping experiment. The density of such defects may be as low as $10^{5} \mathrm{~cm}^{-2}$, which explains the possibility of observing sharp emission lines even in macro-luminescence experiments. Figure 11 schematically illustrates the expected potential shape of a GaAlAs island formed at the GaAs/AlAs interface.

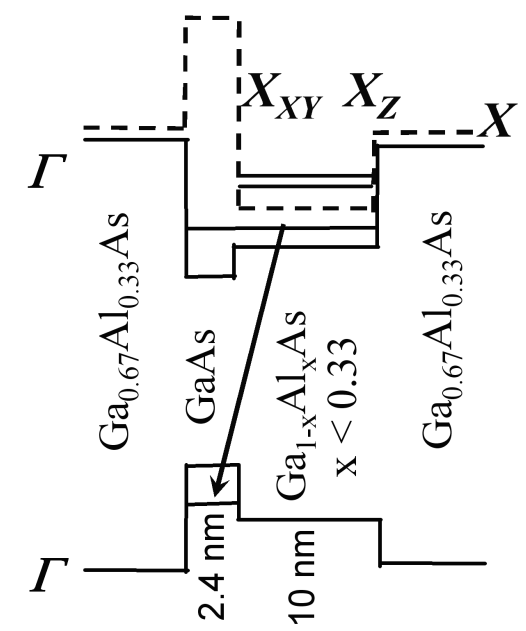

Fig. 11. Schematic representation of the potential distribution in the GaAlAs island formed by Ga diffusion into the AlAs QW.

Because of the very long decay time of the indirect excitons, the $X_{X Y}$ emission is very inefficient and we can easily expect concentrations of photo-excited electron-hole pairs of the order of $10^{10}-10^{11} \mathrm{~cm}^{-2}$ even under weak optical excitation. During their long lifetime, indirect excitons (or spatially separated carriers) will diffuse in the plane of the GaAs/AlAs QW structure until they are trapped into the "easy recombination" GaAlAs island. Such a model employing diffusion of indirect excitons and/or carriers can explain the observed time characteristics of the system. The decay times of the sharp lines (of the order of $\mu \mathrm{s}$ ) are determined by the characteristic time for indirect excitons at the GaAlAs island.

\section{Acknowledgments}

We would like to acknowledge the NRC-Helmholtz joint science and technology fund for support. This work was partially supported by European Committee Projects GMA1-2002-72503 "CEMOS" and RITA-CT-2003-505474 "Access to Research Infrastructure Action of the Improving Human Potential Programme". The Grenoble High Magnetic Field Laboratory is associated with the INPG and Uni- 
versity Joseph Fourier de Grenoble. We are grateful to Z.R. Wasilewski, M.L. Sadowski and B. Jusserand for helpful discussions.

\section{References}

[1] L.V. Butov, A. Zrenner, G. Abstreiter, G. Böhm, G. Weimann, Phys. Rev. Lett. 73, 304 (1994).

[2] A. Zrenner, L.V. Butov, M. Hagn, G. Abstreiter, G. Böhm, G. Weimann, Phys. Rev. Lett. 72, 3382 (1994).

[3] A. Trüby, M. Potemski, R. Planel, Solid State Electron. 40, 139 (1996).

[4] A. Wysmolek, M. Potemski, V. Thierry-Mieg, Physica E 12, 876 (2002).

[5] G. Danan, B. Etienne, F. Mollot, R. Planel, A.M. Jean-Louis, F. Alexandre, B. Jusserand, G. Le Roux, J.Y. Marzin, H. Savary, B. Sermage, Phys. Rev. B 35, 6207 (1987).

[6] H.W. van Kesteren, E.C. Cosman, P. Dawson, K.J. Moore, C.T. Foxon, Phys. Rev. B 39, 13426 (1989) and references therein.

[7] L. Gravier, M. Potemski, A. Fisher, K. Ploog, Solid State Electron. 40, 697 (1996).

[8] M. Sugawara, Y. Nakata, K. Mukai, H. Soji, Phys. Rev. B 55, 13155 (1997).

[9] Q. Wu, R.D. Grober, D. Gammon, D.S. Katzer, Phys. Rev. Lett. 83, 2652 (1999); Q. Wu, R.D. Grober, D. Gammon, D.S. Katzer, Phys. Rev. B 62, 13022 (2000).

[10] K. Brunner, G. Abstreiter, G. Böhm, G. Tränkle, G. Weimann, Phys. Rev. Lett. 73, 1138 (1994); K. Brunner, G. Abstreiter, G. Böhm, G. Tränkle, G. Weimann, Appl. Phys. Lett. 64, 3320 (1994).

[11] D. Gammon, E.S. Snow, D.S. Katzer, Appl. Phys. Lett. 67, 2391 (1995).

[12] A. Babinski, S. Raymond, Z. Wasilewski, J. Lapointe, M. Potemski, Acta Phys. Pol. 105, 547 (2004).

[13] J.G. Tischler, A.S. Bracker, D. Gammon, D. Park, Phys. Rev. B 66, 081310(R) (2002).

[14] K. Matsuda, T. Saiki, S. Nomura, M. Mihara, Y. Aoyagi, S. Nair, T. Takagahara, Phys. Rev. Lett. 91, 177401 (2003).

[15] B. Chwalisz, A. Wysmolek, R. Stepniewski, A. Babinski, M. Potemski, V. Thierry-Mieg, to be published in International Journal of Modern Physics B.

[16] D. Gammon, B.V. Shanabrook, D.S. Katzer, Appl. Phys. Lett. 57, 2710 (1990); D. Gammon, B.V. Shanabrook, D.S. Katzer, Phys. Rev. Lett. 67, 1547 (1991).

[17] K. Leosson, J.R. Jensen, W. Langbein, J.M. Hvam, Phys. Rev. B 61, 10322 (2000).

[18] B. Lita, S. Ghaisas, R.S. Goldman, M.R. Melloch, Appl. Phys. Lett. 75, 4082 (1999).

[19] J. Behrend, M. Wassermeier, W. Braun, P. Krispin, K.H. Ploog, Phys. Rev. B 53, 9907 (1996).

[20] W. Braun, A. Trampert, L. Däweritz, K.H. Ploog, Phys. Rev. B 55, 1689 (1997).

[21] J.-I. Kasai, M. Kawata, Appl. Phys. Lett. 73, 2012 (1998).

[22] M.E. Hoenk, K.J. Vahala, Appl. Phys. Lett. 53, 2062 (1988). 